\title{
Asymptotical forms of canonical mappings near separatrix of Hamiltonian systems
}

\author{
S. S. Abdullaev \\ Institut für Plasmaphysik, Forschungszentrum Jülich GmbH, EURATOM Association, Trilateral Euregio Cluster, \\ D-52425 Jülich, Germany
}

(Received 19 May 2005; published 4 October 2005)

\begin{abstract}
Asymptotical behavior of canonical mappings near the separatrix of Hamiltonian systems subjected to time-periodic perturbations is studied. Based on general forms of these mappings [S. S. Abdullaev, Phys. Rev. E 70, 046202 (2004)] it is shown that the Melnikov-type integrals determining their generating functions can be presented as a sum of regular, $R^{(r e g)}(h)$, and oscillatory, $R^{(o s c)}(h)$, parts. General asymptotical formulas for $R^{(\text {osc })}(h)$ are derived. The oscillatory parts have zeros at primary resonant values of energy. Conditions are found at which the oscillatory parts, $R^{(o s c)}(h)$, can be neglected in the generating functions thus allowing us to obtain simplified mappings depending only the regular parts $R^{(r e g)}(h)$. Since the latter are smooth functions of energy $h$ this allows us also to justify the widely used conventional separatrix mapping determined by $R^{\text {(reg })}$ $\times(h)$ at the separatrix $h=0$. A theory is illustrated for a specific example of a Hamiltonian system, a particle dynamics in periodically perturbed double-well potential.
\end{abstract}

DOI: 10.1103/PhysRevE.72.046202 PACS number(s): 05.45.Ac, 45.05.+x, 45.10.Hj, 45.20.Jj

\section{INTRODUCTION}

Separatrix mapping is a powerful tool to study generic features of Hamiltonian systems near the separatrix, particularly, the onset of chaotic motion and the formation of the stochastic layer [1-4]. It is a map of energy and time variables to certain sections of the phase space of the system. The conventional method to derive separatrix mappings is based on the calculations of the increments of time and energy variables over phase rotation in phase space (see, for instance, Ref. [2]). Particularly, the calculation of energy increment is reduced to the Melnikov integral, i.e., to the integral from the perturbation functions taken along the unperturbed separatrix [5].

The separatrix mapping has been successfully used in various problems of physics and dynamical astronomy to study a chaotic transport of passive particles in structured fluids, magnetic field lines in magnetically confined plasmas, etc. (see Ref. [6] for references). In spite of such numerous applications of the separatrix mapping no rigorous derivation of this mapping has been given, nor has its justification been discussed in the literature. In the previous paper [6] a first step toward mathematically rigorous construction of mappings near the separatrix of Hamiltonian systems has been made. Based on the method of canonical transformation of variables to construct mappings in Hamiltonian systems proposed in Ref. [7], the general procedures to construct canonical mappings near the separatrix have been proposed and their different forms are obtained.

The present work is devoted to study the asymptotical forms of the mappings obtained in Ref. [6] and justification of the separatrix mapping. First we obtain general asymptotical formulas for the Melnikov-type integrals, $R(h)$ near the separatrix which determine the generating functions of mappings. They can be presented as a sum of two parts: a regular part, $R^{(r e g)}(h)$, and an oscillatory part, $R^{(o s c)}(h)$. It is shown that the regular part, $R^{(r e g)}(h)$, is a smooth function of energy $h$, and it is related to the Fourier components, $H_{m n}(h)$, of the perturbation Hamiltonian taken at the resonant values of energy, $h_{m n}$, determined by the primary resonance conditions, $m \omega\left(h_{m n}\right)=\Omega$, where $m \omega(h)$ is a frequency of unperturbed motion, $\Omega$ is a perturbation frequency, and $m$ is an integer number. The oscillatory part, $R^{(o s c)}(h)$, is a fast-oscillating function of $h$ with zeros at the primary resonant values, $h_{m n}$. Since the primary resonances mostly affect the system the oscillatory integrals, $R^{(o s c)}(h)$, in the generating functions can be neglected at low and moderately high perturbation frequencies $\Omega$. This approximation, called primary resonant approximation, allows us to simplify the mapping, and moreover, to justify the validity of the separatrix mapping near the separatrix where $R^{(r e g)}(h)$ can be replaced by the Melnikov integral $R^{(r e g)}(0)$ taken along the unperturbed separatrix.

The paper is organized as follows. In Sec. II we recall the general forms of canonical mappings obtained in Ref. [6]. The structure of the Melnikov-type integrals and their properties are studied in Sec. III. Particularly, the derivation of asymptotical formulas for the Melnikov-type integrals are given in the Appendix. Simplification of mappings near the separatrix and the justification of the separatrix mapping are discussed in Sec. IV. The application of the mapping to the specific Hamiltonian system, namely, to study the dynamics of a particle in a periodically driven double-well potential is given in Sec. V. The obtained results are summarized in the conclusive Sec. VI.

\section{CANONICAL MAPPINGS NEAR SEPARATRIX}

Consider a generic one-degree-of-freedom Hamiltonian system subjected to time-periodic perturbation governed by Hamilton equations

$$
\frac{d x}{d t}=\frac{\partial H}{\partial p}, \quad \frac{d p}{d t}=-\frac{\partial H}{\partial x},
$$

where $(x, p)$ are the canonical coordinate and momentum. The Hamiltonian $H(x, p, t)$ can be presented in the form 


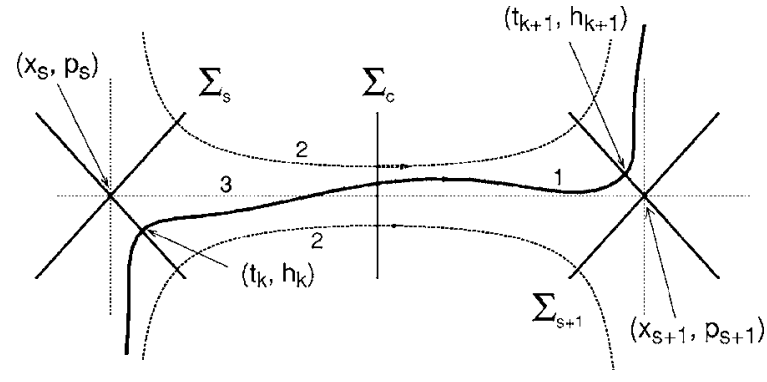

FIG. 1. Schematic view of the mapping. Solid curve describes the perturbed orbit, and dotted curves are the unperturbed orbits near the separatrix.

$$
H(x, p, t)=H_{0}(x, p)+\epsilon H_{1}(x, p, t),
$$

where $H_{0}(x, p)$ is the unperturbed Hamiltonian, $H_{1}(x, p, t)$ is the time-dependent perturbation, $\epsilon$ stands for the small perturbation parameter. Further we consider the multifrequency perturbation with frequencies $\Omega_{n}$ and write the perturbation Hamiltonian $H_{1}(x, p, t)$ as a Fourier series:

$$
H_{1}(x, p, t)=\sum_{n} H_{n}(x, p) \cos \left(\Omega_{n} t+\chi_{n}\right),
$$

where $\chi_{n}$ are the phases of perturbation.

Suppose that the unperturbed system given by Hamiltonian $H_{0}(x, p)$ has one or more saddle points $\left(x_{s}, p_{s}\right)$ $(s=1,2, \ldots)$ at the same energetic level $E$, i.e., $E=H_{0}\left(x_{s}, p_{s}\right)$. These saddle points are connected by phase curves known as separatrices. These curves separate regions of phase space with the different types of motion. Let $\left(x_{s}, p_{s}\right)$ and $\left(x_{s+1}, p_{s+1}\right)$ be two consecutive saddle points with a heteroclinic connection as shown in Fig. 1. If the system has only one saddle point then the points $\left(x_{s}, p_{s}\right)$ and $\left(x_{s+1}, p_{s+1}\right)$ coincide and a saddle-saddle connection is a homoclinic orbit. We put $H$ $=H_{s}=0$ on the separatrix. In Fig. 1 two cross sections $\Sigma_{c}$ and $\Sigma_{s}$ are introduced as follows: the section $\Sigma_{c}$ consists of a segment perpendicular to the separatrix at the midpoint between saddle points, and the section $\Sigma_{s}$ is located near saddle points $\left(x_{s}, p_{s}\right)$ and consists of two segments perpendicular to each other with the crossing point at $\left(x_{s}, p_{s}\right)$. The unperturbed orbits perpendicularly cross these sections.

Let $t_{k}$ and $h_{k}$ be a time instant and an energy of the system when the orbit crosses the section $\Sigma_{s}$, where the index $k$ stands for the iteration number (see Fig. 1). The map along the single saddle-saddle connection,

$$
\left(t_{k+1}, h_{k+1}\right)=\hat{M}_{s+1, s}\left(t_{k}, h_{k}\right),
$$

relates the crossing point $\left(t_{k}, h_{k}\right)$ at the section $\Sigma_{s}$ with the next point $\left(t_{k+1}, h_{k+1}\right)$ at $\Sigma_{s+1}$, as shown schematically in Fig. 1.

The general form of the mapping (4) is obtained in Ref. [6] and in the first order of perturbation parameter $\epsilon$ it has the following symplectic form:

$$
\mathcal{H}_{k}=h_{k}+\epsilon \frac{\partial S^{(k)}\left(t_{k}, \mathcal{H}_{k}\right)}{\partial t_{k}},
$$

$$
\begin{gathered}
\mathcal{T}_{k}=t_{k}-\epsilon \frac{\partial S^{(k)}\left(t_{k}, \mathcal{H}_{k}\right)}{\partial \mathcal{H}_{k}}, \\
\overline{\mathcal{T}}_{k}=\mathcal{T}_{k}+\frac{2 \pi}{\omega\left(\mathcal{H}_{k}\right)}, \\
h_{k+1}=\mathcal{H}_{k}-\epsilon \frac{\partial S^{(k+1)}\left(t_{k+1}, \mathcal{H}_{k}\right)}{\partial t_{k+1}}, \\
t_{k+1}=\overline{\mathcal{T}}_{k}+\epsilon \frac{\partial S^{(k+1)}\left(t_{k+1}, \mathcal{H}_{k}\right)}{\partial \mathcal{H}_{k}},
\end{gathered}
$$

determined by the generating functions

$$
\begin{gathered}
S^{(k)}\left(t_{k}, \mathcal{H}\right)=\sum_{n}\left[K_{n}^{-}(\mathcal{H}) \cos \Phi_{n}^{+}\left(t_{k}, \mathcal{H}\right)-L_{n}^{-}(\mathcal{H}) \sin \Phi_{n}^{+}\left(t_{k}, \mathcal{H}\right)\right] \\
S^{(k+1)}\left(t_{k+1}, \mathcal{H}\right)=-\sum_{n}\left[K_{n}^{+}(\mathcal{H}) \cos \Phi_{n}^{-}\left(t_{k+1}, \mathcal{H}\right)\right. \\
\left.-L_{n}^{+}(\mathcal{H}) \sin \Phi_{n}^{-}\left(t_{k+1}, \mathcal{H}\right)\right] .
\end{gathered}
$$

Here

$$
\Phi_{n}^{ \pm}(t, \mathcal{H})=\Omega_{n}\left(t \pm \frac{\pi}{\omega(\mathcal{H})}\right)+\chi_{n} .
$$

In Eq. (5) $\omega(H)$ is the frequency of motion, determined by the time interval $T(H)$ spent for the phase space point to move from the section $\Sigma_{s}$ to the $\Sigma_{s+1}$ along the unperturbed orbit, i.e., $\omega(H)=2 \pi / T(H)$.

The quantities $K_{n}^{ \pm}(\mathcal{H}), L_{n}^{ \pm}(\mathcal{H})$ are real and imaginary parts of the integral $R_{n}^{ \pm}(\mathcal{H})=K_{n}^{ \pm}(\mathcal{H})+i L_{n}^{ \pm}(\mathcal{H})$, respectively, taken along the unperturbed orbit $x(h, t), p(h, t)$ :

$$
\begin{gathered}
R_{n}^{-}(\mathcal{H})=\int_{-\pi / \omega(\mathcal{H})}^{\tau_{0}-t_{k}-\pi / \omega(\mathcal{H})} V_{n}(\mathcal{H}, \tau) e^{i \Omega_{n} \tau} d \tau, \\
R_{n}^{+}(\mathcal{H})=-\int_{\pi / \omega(\mathcal{H})}^{\tau_{0}-t_{k+1}+\pi / \omega(\mathcal{H})} V_{n}(\mathcal{H}, \tau) e^{i \Omega_{n} \tau} d \tau,
\end{gathered}
$$

where $V_{n}(h, t) \equiv H_{n}(x(h, t), p(h, t))$. We specify the time $t$ in the following way: at $t=0$ the orbit crosses the section $\Sigma_{c}$, and at $t=\mp t_{s}=\mp \pi / \omega(h)$ it crosses the sections $\Sigma_{s}$ and $\Sigma_{s+1}$, respectively. The free timelike parameter $\tau_{0}$ lies in the interval $\left[t_{k}, t_{k+1}\right]$.

If the system has $N_{\text {sep }}$ independent saddle-saddle connections, then there exist $N_{\text {sep }}$ independent mappings (4) which completely determine the dynamics of a Hamiltonian system.

The sequence of mappings $\hat{M}_{s+1, s}$ depends on the topology of saddle-saddle connections in phase space and the trajectory of motion.

The mapping (5) is called symmetric map when the free parameter $\tau_{0}$ is taken exactly in the middle between $t_{k}$ and $t_{k+1}$, i.e., $\tau_{0}=\left(t_{k+1}+t_{k}\right) / 2=t_{k}+\pi / \omega(\mathcal{H})$. Then the integrals $(8)$ take the forms

$$
R_{n}^{ \pm}(\mathcal{H})=\mp \int_{\mp \pi / \omega(\mathcal{H})}^{0} V_{n}(\mathcal{H}, \tau) e^{i \Omega_{n} \tau} d \tau .
$$


The two types of nonsymmetric mappings can be obtained by setting the free time parameter $\tau_{0}$ equal to $\tau_{k+1}$ or $\tau_{k}$. In the first case the mapping (5) is reduced to

$$
\begin{gathered}
h_{k+1}=h_{k}+\epsilon \frac{\partial S\left(t_{k}, h_{k+1}\right)}{\partial t_{k}}, \\
t_{k+1}=t_{k}+\frac{2 \pi}{\omega\left(h_{k+1}\right)}-\epsilon \frac{\partial S\left(t_{k}, h_{k+1}\right)}{\partial h_{k+1}},
\end{gathered}
$$

determined by only one generating function $S\left(t_{k}, h_{k+1}\right)$ :

$$
\begin{aligned}
S\left(t_{k}, h_{k+1}\right)= & \sum_{n}\left[K_{n}\left(h_{k+1}\right) \cos \Phi_{n}^{+}\left(t_{k}, h_{k+1}\right)\right. \\
& \left.-L_{n}\left(h_{k+1}\right) \sin \Phi_{n}^{+}\left(t_{k}, h_{k+1}\right)\right],
\end{aligned}
$$

where $K(h)$ and $L(h)$ are the integrals

$$
K_{n}(h)+i L_{n}(h)=\int_{-\pi / \omega(h)}^{\pi / \omega(h)} V_{n}(h, \tau) e^{i \Omega_{n} \tau} d \tau .
$$

In the second case $\tau_{0}=\tau_{k}$ one obtains

$$
\begin{gathered}
h_{k+1}=h_{k}-\epsilon \frac{\partial S\left(t_{k+1}, h_{k}\right)}{\partial t_{k+1}}, \\
t_{k+1}=t_{k}+\frac{2 \pi}{\omega\left(h_{k}\right)}+\epsilon \frac{\partial S\left(t_{k+1}, h_{k}\right)}{\partial h_{k}},
\end{gathered}
$$

where $S^{(k+1)}\left(t_{k+1}, h_{k}\right)$ is given by

$$
\begin{aligned}
S\left(t_{k+1}, h_{k}\right)= & -\sum_{n}\left[K_{n}\left(h_{k}\right) \cos \Phi_{n}^{-}\left(t_{k+1}, h_{k}\right)\right. \\
& \left.-L_{n}\left(h_{k}\right) \sin \Phi_{n}^{-}\left(t_{k+1}, h_{k}\right)\right] .
\end{aligned}
$$

In a particular case $h=0$ the integrals (12) coincide with the Melnikov integral in the conventional separatrix mapping (see Refs. [2,4]). Indeed, at the limit $h \rightarrow 0$ the frequency $\omega(h) \rightarrow 0$ and the integrals (9) are reduced to

$$
K_{n}(0)+i L_{n}(0)=\int_{-\infty}^{\infty} V_{n}(0, \tau) e^{i \Omega_{n} \tau} d \tau .
$$

\section{STRUCTURE OF MELNIKOV-TYPE INTEGRALS $R_{n}(h)$}

In this section we describe some properties of the Melnikov-type integrals $K_{n}(h)$ and $L_{n}(h)$ defined by Eqs. (9) and (12). First of all consider the relation between coefficients $H_{m n}(h)$ of the perturbation in a Fourier series in action-angle variable $(I, \vartheta)$, i.e.,

$$
\begin{gathered}
H_{1}(h, \vartheta, t)=\sum_{n} H_{n}(h, \vartheta) \cos \left(\Omega_{n} t+\chi_{n}\right), \\
H_{n}(h, \vartheta)=\operatorname{Re} \sum_{m} H_{m n}(h) e^{i m \vartheta}, \\
H_{m n}^{*}(h)=H_{-m, n}(h), \quad H_{m n}(h) \equiv H_{m n}(I(h)),
\end{gathered}
$$

and the integrals $R_{n}(h)=K_{n}(h)+i L_{n}(h)$. According to Eq. (12) one obtains

$$
\begin{aligned}
R_{n}(h) & =\int_{-\pi / \omega(h)}^{\pi / \omega(h)} H_{n}(h, \vartheta) e^{i \Omega_{n} \tau} d \tau \\
& =\operatorname{Re} \sum_{m} H_{m n}(h) \int_{-\pi / \omega(h)}^{\pi / \omega(h)} e^{i\left(m \omega+\Omega_{n}\right) \tau} d \tau \\
& =\frac{2 \pi}{\omega(h)} \sum_{m} \frac{\sin \left\{\pi\left[m-\Omega_{n} / \omega(h)\right]\right\}}{\pi\left[m-\Omega_{n} / \omega(h)\right]} H_{m n}^{*}(h) .
\end{aligned}
$$

As seen from Eq. (17) at the values $h=h_{m n}$ of primary resonances, i.e., $m \omega\left(h_{m n}\right)=\Omega_{n}$, the integral $R_{n}(h)$ is determined by Fourier coefficients $H_{m n}^{*}(h)$, i.e.,

$$
R_{n}\left(h_{m n}\right)=\frac{2 \pi}{\omega(h)} H_{m n}^{*}\left(h_{m n}\right)=\frac{2 \pi m}{\Omega_{n}} H_{m n}^{*}\left(h_{m n}\right) .
$$

The analytical calculation of the integrals $R_{n}(h)$ is not straightforward. The asymptotical method to estimate these integrals is presented in the Appendix. It is shown that the integral $R_{n}(h)$ can be presented as a sum of regular and oscillatory parts,

$$
R_{n}(h)=R_{n}^{(r e g)}(h)+R_{n}^{(o s c)}(h) .
$$

The regular part, $R^{(\mathrm{reg})}(h)$, is a smooth and slowly varying function of the relative energy $h$. We construct this function by extending the function $R_{n}\left(h_{m n}\right)$ [Eq. (18)] defined at discrete resonant values of $h_{m n}$ (or $m$ ) to continuous values of $h$ by replacing the discrete mode number $m$ by the continuous one $m=\Omega_{n} / \omega(h)$, i.e.,

$$
R_{n}^{(r e g)}(h)=\frac{2 \pi}{\omega(h)} H_{\Omega_{n} / \omega(h), n}^{*}(h) .
$$

At the limit $|h| \rightarrow 0$ it tends to the value $R(0)$, i.e., to the Melnikov integrals (15). Analytical and numerical calculations of $R^{(r e g)}(h)$ for typical Hamiltonian systems presented in Sec. V show that $R^{(r e g)}(h)$ is sufficiently close to $R(0)$ at a certain small region near the separatrix $h=0$, i.e., $R^{(r e g)}(h)$ $\approx R(0)$.

The oscillatory part, $R^{(o s c)}(h)$, is a fast-oscillating function of $h$ with a vanishing amplitude at the limit $|h| \rightarrow 0$ : $R^{(o s c)}(h) \rightarrow 0$. The asymptotical formulas for $R^{(o s c)}(h)$ at small values of $h$ are given in the Appendix. For Hamiltonian systems with hyperbolic saddle points they have generic features near the separatrix. It was shown that for the perturbation Hamiltonian, $H_{1}(x, p, t)$, with the nonvanishing first derivative at the saddle point, $\left(x_{s}, p_{s}\right)$, i.e., $\partial H_{1}(x, p, t) / \partial x$ $\neq 0$ [or $\left.\partial H_{1}(x, p, t) / \partial p \neq 0\right]$, the leading term of $R^{(o s c)}(h)$ has the following asymptotics:

$$
R^{(o s c)}(h) \sim \sqrt{|h|} \begin{cases}\sin \left(\frac{\pi \Omega_{n}}{\omega(h)}\right), & \text { for } h<0, \\ \cos \left(\frac{\pi \Omega_{n}}{\omega(h)}\right), & \text { for } h>0 .\end{cases}
$$

Since $\omega(h) \rightarrow 0$ when $|h| \rightarrow 0$ the frequency of oscillations of $R^{(o s c)}(h)$ in $h$ increases with approaching the separatrix. According to the definitions of $R^{(\text {reg })}(h)$ and $R^{(o s c)}(h)$ given by Eqs. (19) and (20) and the property (18) the function 
$R^{(o s c)}(h)$ has zeros at the primary resonance values of $h$ $=h_{m n}$ when $m \omega\left(h_{m n}\right)=\Omega_{n}$.

Moreover, the integrals $R^{(o s c)}(h)$ have a rescaling invariance property near the separatrix,

$$
R^{(o s c)}\left(\lambda^{2} h\right)=\lambda R^{(o s c)}(h),
$$

where $\lambda=\exp \left(2 \pi \gamma / \Omega_{n}\right)$ is the universal rescaling parameter (see, e.g., Ref. [8]). This property follows from the universal logarithmic asymptotics, $\omega(h) \sim 2 \pi \gamma / \ln |h|$, of the frequency of motion $\omega(h)$ near the separatrix of Hamiltonian systems with hyperbolic saddle points. Here $\gamma$ is a growth increment of orbits.

Complete asymptotical formulas for the integrals $R^{(o s c)}(h)$ for generic Hamiltonian systems are derived in the Appendix. We shall also study the properties of these integrals in Sec. V for a specific Hamiltonian system.

We conclude this section with a note that the relation (18) between the Fourier coefficients, $H_{m n}(h)$, of the perturbation Hamiltonian $H_{1}(h, \vartheta, t)$ and the regular part, $R_{n}^{(\text {reg })}(h)$, of the Melnikov-type integral allows one to rewrite the well-known formula for the width of resonances, $W_{m n}$, through a single function $R^{(r e g)}(h)$, i.e.,

$$
W_{m n}=4\left|\frac{\epsilon H_{m n}\left(h_{m n}\right)}{\omega_{I}\left(h_{m n}\right)}\right|^{1 / 2}=4\left|\frac{\epsilon R_{n}\left(h_{m n}\right)}{2 \pi \omega_{h}\left(h_{m n}\right)}\right|^{1 / 2},
$$

where $\omega_{I}(h) \equiv d \omega / d I, \omega_{h}(h) \equiv d \omega / d h$.

\section{SIMPLIFICATION OF MAPPINGS}

The symmetric mapping (5) with the generating functions (6), as well as the nonsymmetric mappings (10) and (13) determined by generating functions (11) and (14), respectively, have a rather complicated structure due to presence of oscillatory parts of integrals $R_{n}(h)$ in the generating functions $S(h, t)$. This may cause some difficulties in numerical solutions of implicit equations in the mappings, especially when $h$ approaches 0. For this reason it is desirable to simplify mappings. Below we consider such an approximation which would not only simplify mappings, but also justify the separatrix mappings.

\section{A. "Primary resonant" approximation}

As has been noted above, the oscillatory parts of the integrals $R_{n}(h)$ have zeros at primary resonant values of $h$ $=h_{m n}$, where $R_{n}\left(h_{m n}\right)$ is proportional to Fourier coefficients $H_{m n}$ of the perturbation Hamiltonian $H_{1}(I, \vartheta, t)$ [see Eq. (18)]. The primary resonant perturbation, $H_{m n} \cos \left(m \vartheta-\Omega_{n} t\right)$, significantly affects the system near the resonant values of $h_{m n}\left[m \omega\left(h_{m n}\right)=\Omega_{n}\right]$, while the effect of other nonresonant terms is negligible. Then near the resonant values of $h_{m n}$ the oscillatory part $R_{n}^{(o s c)}(h)$ is significantly smaller than the regular part $R_{n}^{(\text {reg })}(h)$ :

$$
\left|R_{n}^{(o s c)}(h)\right| \ll\left|R_{n}^{(r e g)}(h)\right|, \quad \text { for } h \approx h_{m n} .
$$

Then one can neglect the oscillatory parts, $R_{n}^{(o s c)}(h)$, in the generating functions, $S(h, t)$, replacing the integrals $R_{n}(h)$ by their regular parts $R_{n}^{(\text {reg })}(h)$. Furthermore, we shall call this approximation a "primary resonant" approximation.

\section{B. Simplified form of mappings}

Further simplification of the mappings can be done using the smallness of perturbation parameter $\epsilon$. Eliminating the intermediate variables, $\mathcal{H}, \mathcal{T}$, we transform a set of Eq. (5) into

$$
\begin{gathered}
h_{k+1}=h_{k}-\epsilon\left(\frac{\partial S_{k+1}}{\partial t_{k+1}}-\frac{\partial S_{k}}{\partial t_{k}}\right), \\
t_{k+1}=t_{k}+\frac{2 \pi}{\omega\left(\mathcal{H}_{k}\right)}+\epsilon\left(\frac{\partial S_{k+1}}{\partial \mathcal{H}_{k}}-\frac{\partial S_{k}}{\partial \mathcal{H}_{k}}\right) .
\end{gathered}
$$

Using Eqs. (5) and (6), one can show that

$$
\begin{aligned}
\frac{2 \pi}{\omega\left(\mathcal{H}_{k}\right)}+\epsilon\left(\frac{\partial S_{k+1}}{\partial \mathcal{H}_{k}}-\frac{\partial S_{k}}{\partial \mathcal{H}_{k}}\right)= & \frac{\pi}{\omega\left(h_{k}\right)}+\frac{\pi}{\omega\left(h_{k+1}\right)}+G\left(t_{k}, h_{k+1}, h_{k}\right) \\
& +O\left(\epsilon^{2}\right), \\
\epsilon\left(\frac{\partial S_{k+1}}{\partial t_{k+1}}-\frac{\partial S_{k}}{\partial t_{k}}\right)= & \epsilon F\left(t_{k}, h_{k+1}, h_{k}\right)+O\left(\epsilon^{2}\right),
\end{aligned}
$$

where

$$
\begin{aligned}
F\left(t_{k}, h_{k+1}, h_{k}\right)= & \sum_{n} \Omega_{n}\left[K_{n}^{(\text {reg })}\left(h_{k+1}\right) \sin \Phi_{n}^{+}\left(t_{k}, h_{k}\right)+L_{n}^{(\text {reg })}\right. \\
& \left.\times\left(h_{k}\right) \cos \Phi_{n}^{+}\left(t_{k}, h_{k}\right)\right] \\
G\left(t_{k}, h_{k+1}, h_{k}\right)= & -\sum_{n}\left(\frac{d K_{n}^{(r e g)}\left(h_{k+1}\right)}{d h_{k+1}} \cos \Phi_{n}^{+}\left(t_{k}, h_{k}\right)\right. \\
& \left.-\frac{d L_{n}^{(r e g)}\left(h_{k+1}\right)}{d h_{k+1}} \sin \Phi_{n}^{+}\left(t_{k}, h_{k}\right)\right),
\end{aligned}
$$

with the coefficients $K_{n}^{(r e g)}(h), L_{n}^{(r e g)}(h)$ are regular parts of the integrals (12). Then neglecting the terms of order $\epsilon^{2}$ we obtain

$$
h_{k+1}=h_{k}-\epsilon F\left(t_{k}, h_{k+1}, h_{k}\right) \text {, }
$$

$$
t_{k+1}=t_{k}+\frac{\pi}{\omega\left(h_{k}\right)}+\frac{\pi}{\omega\left(h_{k+1}\right)}+\epsilon G\left(t_{k}, h_{k+1}, h_{k}\right) .
$$

It is easy to check that the mapping (27) is area-preserving, i.e., $\operatorname{det}\left|\partial\left(h_{k+1}, t_{k+1}\right) / \partial\left(h_{k}, t_{k}\right)\right|=1$, and invariant with respect to the time reversing transformation, $k \leftrightarrow k+1$.

\section{Separatrix mapping approximation}

For typical Hamiltonian systems the regular part, $R_{n}^{(r e g)}(h)$, is a smooth function of $h$ and its deviation from $R_{n}^{(r e g)}(0)$ is small. Then the integrals (9) in the generating functions (6) can be replaced by the Melnikov-type integrals (15), i.e., $K_{n}(h)=K_{n}(0), L_{n}(h)=L_{n}(0)$. Then the mapping (27) can be further simplified to 


$$
\begin{gathered}
h_{k+1}=h_{k}-\epsilon \sum_{n} \Omega_{n} \times\left\{K_{n}(0) \sin \left[\Omega_{n}\left(t_{k}+\frac{\pi}{\omega\left(h_{k}\right)}\right)+\chi_{n}\right]\right. \\
\left.+L_{n}(0) \cos \left[\Omega_{n}\left(t_{k}+\frac{\pi}{\omega\left(h_{k}\right)}\right)+\chi_{n}\right]\right\} \\
t_{k+1}=t_{k}+\frac{\pi}{\omega\left(h_{k}\right)}+\frac{\pi}{\omega\left(h_{k+1}\right)},
\end{gathered}
$$

where $K_{n}(0)$ and $L_{n}(0)$ are the integrals defined by Eq. (15). The mapping of type (28) was obtained in Refs. [9,10] from the conventional separatrix mapping by shifting the time $t_{k}$ from section $\Sigma_{c}$ to section $\Sigma_{s}$.

Since both variables $(t, h)$ in the mappings (27) and (28) are defined in the neighborhood of the saddle points, they become important to study the dynamics and statistical properties of chaotic motion in a system. This is because of the fact that trajectories spend most of time near the saddle point, and therefore the whole dynamics is mainly determined by the phase space structure of the system in the neighborhood of saddle points.

\section{On the validity of the primary resonant approximation}

In this approximation one neglects the oscillatory parts $R_{n}^{(o s c)}(h)$ of the integrals $R_{n}(h)$ retaining only the smooth regular parts $R_{n}^{(r e g)}(h)$. This procedure is equivalent to taking into account the effect of only primary resonances of type $m \omega\left(h_{m n}\right)=\Omega_{n}$, near which the oscillatory functions $R_{n}^{(o s c)}(h)$ are negligibly small in comparison with $R_{n}^{(r e g)}(h)$ [see Eq. (24)]. This condition is satisfied for the low and moderately high frequencies of perturbation $\Omega_{n}$, when $R_{n}^{(r e g)}(h)$, particularly, $R_{n}^{(r e g)}(0)$, is not negligibly small.

However, for the large perturbation frequencies, $\Omega_{n}$, the condition (24) may be violated. At the large $\Omega_{n}$ the integrals $R_{n}^{(r e g)}(h)$, as well as $R_{n}^{(r e g)}(0)$, are exponentially small in the large interval of $h$ near the separatrix. Then the effect of the primary resonances, $m \omega(h)=\Omega_{n}$, on the system becomes negligibly small. In this case the nonprimary resonances of type $m \omega\left(h_{m n}\right)=s \Omega_{n}(s>1)$, at which $\left|R_{n}^{(o s c)}\left(h_{m n}\right)\right| \gg\left|R_{n}^{(r e g)}(h)\right|$, may affect the system although the corresponding perturbation terms $H_{m n}(I)$ in the Fourier expansion of $H_{1}(I, \vartheta, t)$ are exponentially small.

However, the detailed study of the validity of the "primary resonant approximation" requires a separate investigation which, probably, should take into account the higher order terms of the generating function $S(t, h)$ in a perturbative series of powers of perturbation parameter $\epsilon$.

\section{MOTION IN A PERTURBED DOUBLE-WELL POTENTIAL}

We illustrate the method presented above on the example of a motion of particle in a double-well potential in the presence of external time-periodic perturbation. The system is governed by the Hamiltonian

$$
H=H_{0}(x, p)+\epsilon H_{1}(x, p, t),
$$

(a)

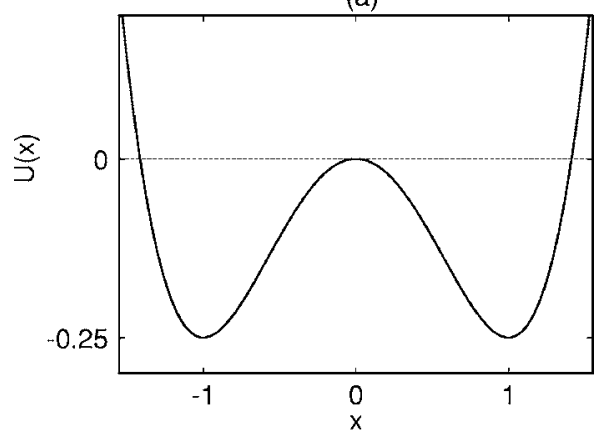

(b)

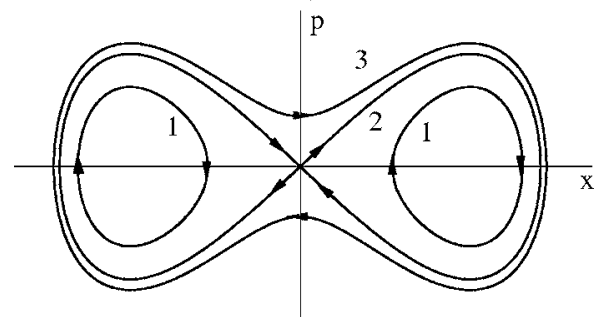

FIG. 2. (a) Double-well potential potential $U(x)=-x^{2} / 2+x^{4} / 4$. (b) Phase space of motion.

$$
\begin{gathered}
H_{0}(x, p)=\frac{p^{2}}{2}-\frac{x^{2}}{2}+\frac{x^{4}}{4}, \\
H_{1}(x, p, t)=\epsilon x \cos (\Omega t+\chi),
\end{gathered}
$$

where $\epsilon$ stands for the small perturbation amplitude. Figure 2 shows the potential function $U(x)=-x^{2} / 2+x^{4} / 4$ (a), and the phase space of unperturbed motion $(\epsilon \equiv 0)$ (b). It has two elliptic fixed points at $(x= \pm 1, p=0)$ and one hyperbolic fixed point at $(x=0, p=0)$. A particle is trapped in one of potential wells (curves 1) at $-1 / 4<H<0$ [curves 1 in Fig. 2(b)], and it is untrapped at $H>0$ (curve 3 ). At the separatrix $H=0$ (curve 2). The unperturbed trajectory $x(t), p(t)$ for the trapped motion $(H<0)$ is given by

$$
\begin{gathered}
x(t)= \pm a \operatorname{dn}(u ; k), \\
p(t)=\mp \frac{a^{2} k^{2}}{\sqrt{2}} \operatorname{sn}(u ; k) \operatorname{cn}(u ; k), \\
u=a t / \sqrt{2}, \quad a=\sqrt{1+\sqrt{1+4 H}},
\end{gathered}
$$

where $\operatorname{sn}(u ; k), \operatorname{cn}(u ; k), \operatorname{dn}(u ; k)$ are the Jacobi elliptic functions with a module $k$,

$$
k=\frac{\sqrt{2}(1+4 H)^{1 / 4}}{(1+\sqrt{1+4 H})^{1 / 2}} .
$$

The unperturbed frequency, $\omega(H)=d H_{0}(I) / d I$, of this motion is 


$$
\omega(H)=\frac{\pi a}{\sqrt{2} K(k)} .
$$

Particle orbits outside the potential wells $(H>0)$ are described by

$$
\begin{gathered}
x(\vartheta)= \pm a \operatorname{cn}(u ; 1 / k), \\
p(\vartheta)=\mp a(1+4 H)^{1 / 4} \operatorname{sn}(u ; 1 / k) \sqrt{1-k^{-2} \operatorname{sn}^{2}(u ; 1 / k)}, \\
u=a k t / \sqrt{2},
\end{gathered}
$$

and the frequency is given by

$$
\omega(H)=\frac{\pi k a}{\sqrt{2} K\left(k^{-1}\right)} .
$$

Near the separatrix $(H \rightarrow 0)$ the frequency, $\omega(h)$, goes to zero according to the following asymptotics:

$$
\omega(H)=\frac{1}{\ln \frac{16}{|H|}}+O(H), \quad|H| \rightarrow 0 .
$$

Using Eqs. (30) and (32) the perturbation Hamiltonian $H_{1}(x, p, t)$ in Eq. (29) can be expanded in Fourier series in the angle variable $\vartheta$ :

$$
\begin{aligned}
H_{1}(I, \vartheta, t)= & \sum_{s=-1,1} \sum_{m=1}^{\infty} H_{m}(H) \\
& \times \begin{cases}\cos (m \vartheta-s \Omega t), & \text { for } H<0, \\
\cos ([m-1 / 2] \vartheta-s \Omega t), & \text { for } H>0,\end{cases}
\end{aligned}
$$

where

$$
\begin{aligned}
& H_{m}(H)= \pm a \times \begin{cases}\frac{\pi}{K(k)} \frac{q_{-}^{m}}{1+q_{-}^{2 m}}, & \text { for } H<0 \\
\frac{\pi}{K\left(k^{-1}\right)} \frac{q_{+}^{m-1 / 2}}{1+q_{+}^{2 m-1}}, & \text { for } H>0,\end{cases} \\
& q_{-}=\exp \left[-\pi K\left(\sqrt{1-k^{2}}\right) / K(k)\right], \\
& q_{+}=\exp \left[-\pi K\left(\sqrt{1-k^{-2}}\right) / K\left(k^{-1}\right)\right] .
\end{aligned}
$$

From Eq. (35) follows that the primary resonance conditions are $m \omega(H)=\Omega(m=0,1,2, \ldots)$ for the trapped motion $(H<0)$. For the untrapped motion $(H>0)$ the corresponding conditions are $(2 m-1) \omega(H)=2 \Omega$.

The geometrical scheme of the mapping is given in Fig. 3 . The section $\Sigma_{s}$ located near the saddle point $\left(x_{s}=p_{s}=0\right)$ consists of two perpendicular segments of the $x$ and $p$ axes with the center at $\left(x_{s}=0, p_{s}=0\right)$. The section $\Sigma_{c}$ consists of the segment of the $x$ axis located near the farthest crossing points of the unperturbed separatrix with the $x$ axis. Two saddlesaddle connections are located in the right $(x>0)$ and left $(x<0)$ halves of phase space $(x, p)$, respectively.

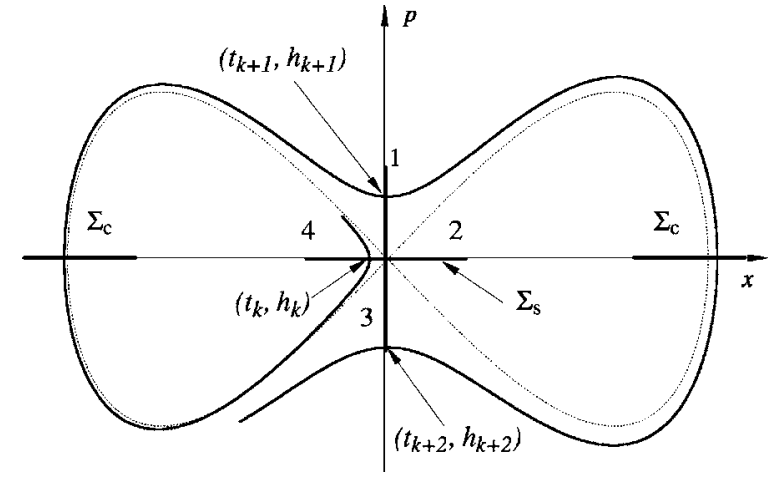

FIG. 3. Geometry of the separatrix map. The solid curve describes the perturbed orbit, and the dotted curve is the unperturbed separatrix.

According to Eq. (29) the perturbation function $V_{n}(\mathcal{H}, \tau)$ $=x(\tau)$, and using Eqs. (30) and (32) for $x(\tau)$ the integral $K(h)$ [Eq. (12)] is reduced to

$$
\begin{aligned}
K(h)= & \int_{-\pi / \omega(h)}^{\pi / \omega(h)} x^{( \pm)}(\tau) \cos (\Omega \tau) d \tau= \pm \frac{a \pi}{\omega(h)} \int_{-1}^{1} \\
& \times \begin{cases}\operatorname{dn}(K(k) \tau ; k) \cos (\Omega \pi \tau / \omega) d \tau, & \text { for } h<0, \\
\operatorname{cn}(K(1 / k) \tau ; 1 / k) \cos (\Omega \pi \tau / \omega) d \tau, & \text { for } h>0 .\end{cases}
\end{aligned}
$$

The signs $( \pm)$ stand for the right $(x>0)$ and left $(x<0)$ halves of phase space, respectively. At the separatrix $h=0$ we have

$$
\begin{aligned}
K(0) & =\int_{-\infty}^{\infty} x_{s}^{( \pm)}(t) \cos (\Omega \tau) d \tau= \pm \sqrt{2} \int_{-\infty}^{\infty} \frac{\cos (\Omega \tau) d \tau}{\cosh \tau} \\
& =\frac{ \pm \sqrt{2} \pi}{\cosh (\pi \Omega / 2)}
\end{aligned}
$$

Since $x(t)=x(-t)$ one can show that $L(h) \equiv 0$.

As was shown in Sec. III the integral $K(h)$ consists of regular and oscillatory parts: $K(h)=K^{(r e g)}(h)+K^{(o s c)}(h)$. According to Eq. (20) the regular part can be expressed through the Fourier components $H_{m}(H)$ [Eq. (36)] of the perturbation Hamiltonian. Using Eqs. (31) and (33) we have

$$
K^{(r e g)}(h)=\frac{2 \pi}{\omega(h)} H_{\Omega / \omega(h)}(h)= \pm \frac{\pi \sqrt{2}}{\cosh \left[\sqrt{2} \Omega K\left(\sqrt{1-k^{2}}\right) / a\right]}
$$

for the case $h<0$, and

$$
\begin{aligned}
K^{(\text {reg })}(h) & =\frac{2 \pi}{\omega(h)} H_{\Omega / \omega(h)+1 / 2}(h) \\
& = \pm \frac{\pi \sqrt{2}}{k \cosh \left[\sqrt{2} \Omega K\left(\sqrt{1-k^{-2}}\right) / k a\right]}
\end{aligned}
$$

for the case $h>0$. In the limit $|h| \rightarrow 0$ the both expressions of $K^{(r e g)}(h)$ coincide with $K(0)[$ Eq. (38)] obtained by the direct integration. 
The asymptotical formula for $K^{(o s c)}(h)$ can be found using the general asymptotical formulas for the Melnikov-type integrals, $R^{(o s c)}(h)$, near the separatrix obtained in the Appendix. Our problem corresponds to cases (ii) and (iii) considered in the Appendix, Sec. 5, and the expression for $K^{(o s c)}$ $\times(h)$ is given by Eq. (A31). For the Hamiltonian system (29) we have the following parameters: $\alpha=\gamma=1, a_{\xi}=1, a_{\eta}=b_{\xi \xi}$ $=b_{\xi \eta}=b_{\eta \eta}=0$. Therefore one obtains

$$
K^{(o s c)}(h)=\mp \frac{2 \sqrt{2|h|}}{\Omega^{2}+1} \times \begin{cases}\Omega \sin [\pi \Omega / \omega(h)], & \text { for } h<0, \\ -\cos [\pi \Omega / \omega(h)], & \text { for } h>0,\end{cases}
$$

where the upper sign (-) corresponds to the right side of the phase space $(x>0)$, and the lower sign $(+)$ - to the left side of the phase space $(x<0)$.

Figure 4 shows the dependence of the integral $K(h)$ on $h$ obtained by the direct numerical integration of Eq. (37), as well as by the analytical formulas (39)-(41): solid curve 1 corresponds to the numerical calculations, dashed curve 2 corresponds to the analytical result, and dotted curve 3 corresponds to the regular part $K^{(r e g)}(h)$ given by Eqs. (39) and (40). The perturbation frequency is taken equal to $\Omega$ $=4.53236$. The corresponding rescaling parameter $\lambda$ $=\exp (2 \pi \gamma / \Omega)$ is equal to 4 .

As seen from Figs. 4(a)-4(c), analytical formulas (39) and (40), the regular part $K^{(r e g)}(h)$ and the asymptotical formula (41) for the oscillatory part, $K^{(o s c)}(h)$, well describe the behavior of the integral $K(h)$. The accuracy of approximation increases with approaching the separatrix. On the other hand, the numerical calculations confirm also the following rescaling property:

$$
K^{(o s c)}\left(\lambda^{2} h\right)=\lambda K^{(o s c)}(h)
$$

of the oscillatory part of the integral $K(h)$ following from the asymptotical formula (41).

One can easily see that zeros of $K^{(o s c)}(h)$ coincide with the primary resonant values of $h_{m n}: m \omega(h)=\Omega(m=0,1,2, \ldots)$ at $h<0$, and $(2 m-1) \omega(h)=2 \Omega$ for $h>0$. Therefore according to the primary resonant approximation (see Sec. IV A) in the mapping one can neglect oscillatory parts $K^{(o s c)}(h)$ retaining only smooth regular parts $K^{(r e g)}(h)$.

The dynamics of the system is described by two mappings, $\left(t_{k+1}, h_{k+1}\right)=\hat{M}^{( \pm)}\left(t_{k}, h_{k}\right)$, Eq. (27), corresponding to the two different saddle-saddle connections:

$$
\begin{aligned}
h_{k+1}= & h_{k} \mp \epsilon \Omega K^{(r e g)}\left(h_{k+1}\right) \sin \left(\varphi_{k}+\frac{\pi \Omega}{\omega\left(h_{k}\right)}+\chi\right), \\
\varphi_{k+1}= & \varphi_{k}+\frac{\pi \Omega}{\omega\left(h_{k+1}\right)}+\frac{\pi \Omega}{\omega\left(h_{k}\right)} \mp \epsilon \Omega \frac{d K^{(r e g)}\left(h_{k+1}\right)}{d h_{k+1}} \\
& \times \cos \left(\varphi_{k}+\frac{\pi \Omega}{\omega\left(h_{k}\right)}+\chi\right),
\end{aligned}
$$

where the phase variable $\varphi=\Omega t$ is introduced. The map with the $(+)$ sign describes the right side of phase space $(x>0)$, while one with the $(-)$ sign corresponds to $(x<0)$. (a)

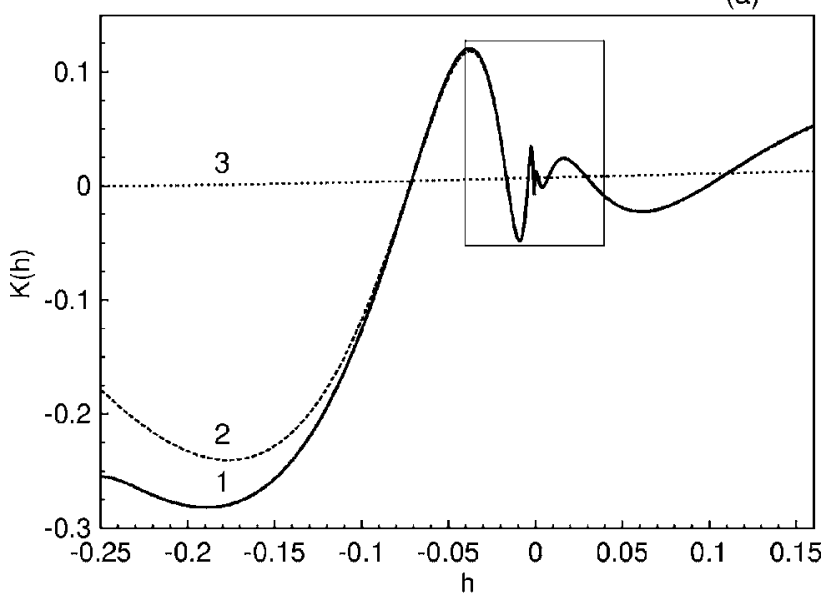

(b)

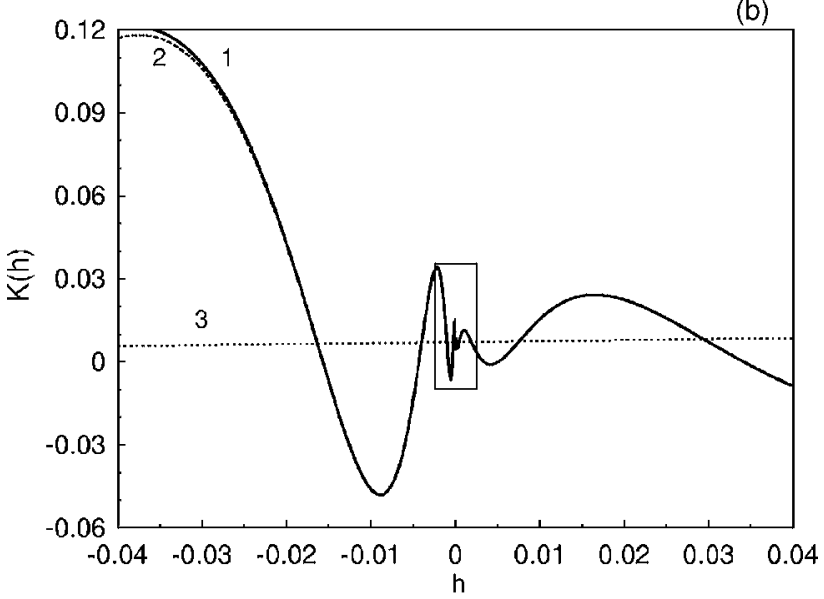

(c)

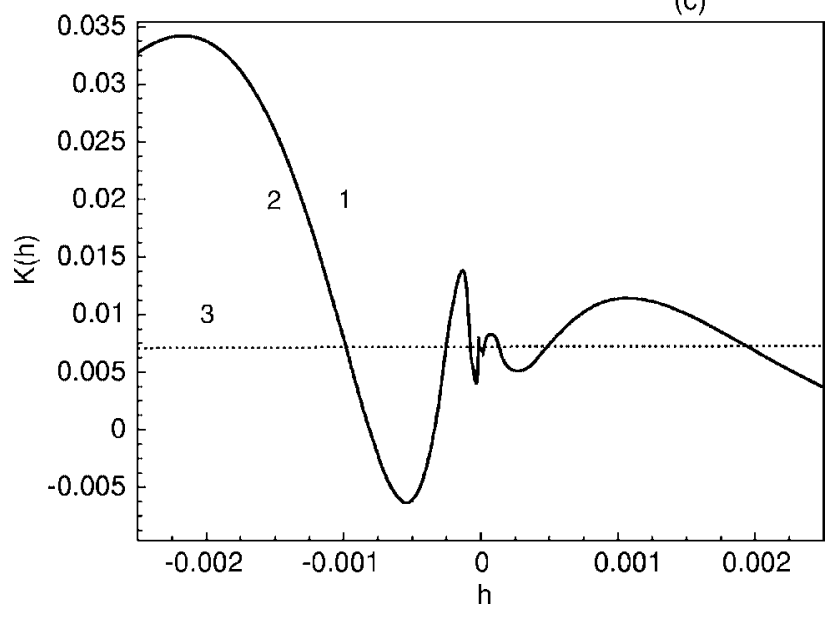

FIG. 4. Dependence of the integral $K(h)$ on the relative energy $h$ : solid curve 1 describes $K(h)$ obtained by the numerical integration of the integral (37), and dashed curve 2 describes $K(h)$ obtained by the analytical formulas (39)-(41), and dotted curve 3 corresponds to the regular part $K^{(r e g)}(h)$ given by Eqs. (39) and (40). (a) In the interval $-0.25<h<0.16$ : (b) expanded view of $K(h)$ in the rectangular box region shown in (a); (c) expanded view of the rectangular box region shown in (b). The perturbation frequency $\Omega=4.53236$, and the rescaling parameter $\lambda=\exp (2 \pi \gamma / \Omega)=4$. 
Further simplification of the mapping can be done near the small neighborhood of the separatrix. Using the asymptotics of the frequency $\omega(h)$ [Eq. (34)] $|h| \rightarrow 0$ and replacing $K^{(r e g)}(h)$ by $K(0)$ we obtain

$$
\begin{gathered}
h_{k+1}=h_{k} \mp \epsilon \Omega K(0) \sin \left(\varphi_{k}+\frac{\Omega}{2} \ln \frac{16}{\left|h_{k}\right|}+\chi\right), \\
\varphi_{k+1}=\varphi_{k}+\frac{\Omega}{2}\left(\ln \frac{16}{\left|h_{k}\right|}+\ln \frac{16}{\left|h_{k+1}\right|}\right) .
\end{gathered}
$$

The dynamics of system near the separatrix is described by the sequence of iterations of the maps $\hat{M}^{( \pm)}$. This sequence is determined by a certain rule given in Ref. [6].

The mappings (43) and (44) are valid for small values of perturbation, $\epsilon \ll 1$. Note that the second mapping (44) is applicable only to the area close to the separatrix, while the first mapping (43) can be applied also far from the separatrix.

The mapping (43) is applied to obtain Poincaré sections of system in the $(\varphi, h)$ plane of the first $(h>0)$ and fourth branches $(h<0)$ of the section $\Sigma_{s}$ (see Fig. 3). It has been also compared with the small step numerical integration of the Hamiltonian system (29) using the symplectic integrator proposed in Ref. [11]. The mapping result is shown in Fig. 5(a), and the results obtained from the numerical integration is presented Fig. 5(b). Calculations in both cases were performed with a set of identical initial coordinates. As seen from Fig. 5 the mapping well reproduces the structure of phase space. For the time step of integration, $\Delta t$, of the equation, equal to $4 \pi \times 10^{-3} / \Omega$, the mapping runs two order faster than the small-step symplectic integrator.

\section{CONCLUSION}

In summary, we have studied the asymptotical behavior of canonical mappings near the separatrix of generic Hamiltonian systems with one degree of freedom in the presence time-periodic perturbations. The canonical mappings of these systems are determined by the Melnikov-type integrals. It is shown that these integrals have a generic asymptotic behavior near the separatrix. They can be presented as a sum of two parts: the regular part, which is a smooth function of the relative energy, and the oscillatory part. The latter is an oscillatory function of energy with zeros at the primary resonant values of energy. This property allows one to neglect the oscillatory parts of the Melnikov integrals in the mappings for the low and medium frequencies of perturbations when the regular part of integrals are not negligibly small. This approximation, called primary resonant approximation, simplifies the mapping. In the regions near the separatrix the smooth regular part of the Melnikov integral can be approximated by its value at the separatrix which thus reduces the mapping to the separatrix mapping. We have illustrated this theory on the example of particles dynamics in a periodically driven double-well potential. The simplified mapping as well as the separatrix mapping well reproduce the results obtained using the small-step symplectic integration of the equations of motion.

The canonical mappings near the separatrix obtained in this work are not directly applicable to the many-degrees-of-
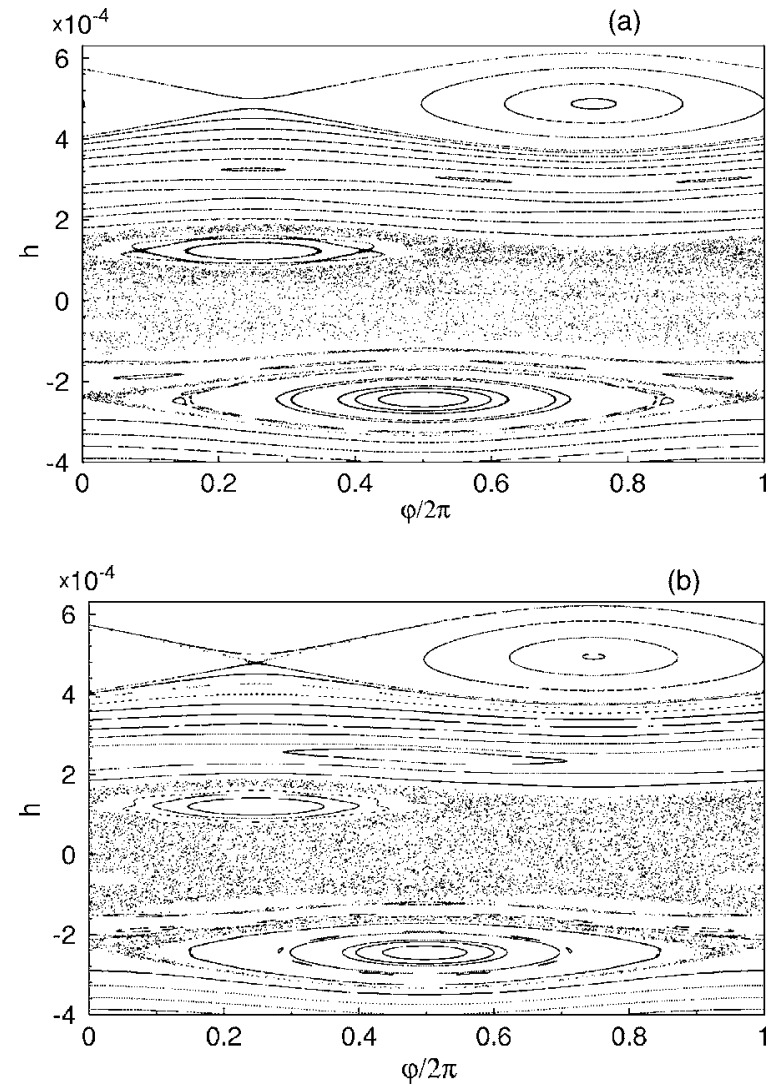

FIG. 5. Poincaré sections to the first and fourth branches of the section $\Sigma_{s}$ : (a) obtained by the mapping (43); (b) by the direct integration of the Hamiltonian system (29). Initial coordinates in both cases are identical. The perturbation frequency $\Omega$ is the same as in Fig. 4, and the perturbation amplitude $\epsilon=10^{-3}$.

freedom Hamiltonian systems. However, the method of canonical transformation of variables to construct mappings near the separatrix given in the previous (see Ref. [6]) and the present works can be generalized to such Hamiltonian systems.

\section{ACKNOWLEDGMENTS}

This work has been partially supported by Project No. SFB 591 of Deutsche Forschungsgemeinschaft (DFG).

\section{APPENDIX: ASYMPTOTIC ESTIMATIONS OF THE INTEGRAL $R_{n}(h)$ NEAR SEPARATRIX}

\section{General structure of integrals}

For simplicity we shall omit the subscripts $n$ in $R_{n}(h)$. Consider three types of the Melnikov-type integrals defined by Eqs. (9) and (12). We study the asymptotics of $R(h)$, $R^{+}(h)$, and $R^{-}(h)$ near the separatrix, i.e., $|h| \ll 1$. They can be presented as a sum of regular, $R^{(r e g)}(h)$, and oscillatory, $R^{(o s c)}(h)$, parts,

$$
\begin{gathered}
R(h)=R^{(r e g)}(h)+R^{(o s c)}(h), \\
R^{ \pm}(h)=R^{(r e g) \pm}(h)+R^{(o s c) \pm}(h),
\end{gathered}
$$

where the regular parts 


$$
\begin{gathered}
R^{(r e g)}(h)=R^{(r e g)+}(h)+R^{(r e g)-}(h), \\
R^{(r e g) \pm}(h)=F(h, \pm 0),
\end{gathered}
$$

and the oscillatory parts

$$
\begin{gathered}
R^{(o s c)}(h)=R^{(o c s)+}(h)+R^{(o c s)-}(h), \\
R^{(o c s) \pm}(h)= \pm F(h, \pm \pi / \omega(h)),
\end{gathered}
$$

are defined through the function $F(h, \tau)$,

$$
F(h, \tau)=\int V(h, \tau) e^{i \Omega \tau} d \tau .
$$

Similar relations can be written for $R^{(\text {reg }) \pm}(h)$ and $R^{(o s c) \pm}(h)$. Further we suppose that the function $V(h, \tau)$ vanishes at the saddle points $\left(q_{s}, p_{s}\right)$, i.e., $V(0, \infty)=H_{1}\left(q_{s}, p_{s}\right)=0$. Then we have $F(0, \infty)=0$. In general cases, this condition can be satisfied by subtracting from the Hamiltonian a term $H_{1}\left(q_{s}, p_{s}, t\right)$ which does not affect the equations of motion.

Below we show that near the separatrix of the Hamiltonian system with hyperbolic fixed points the oscillatory parts of the integrals (12) have a generic asymptotic behavior. In the following sections we derive the asymptotical formulas for $R^{(o s c)}(h), R^{(o s c) \pm}(h)$ in the limit $|h| \rightarrow 0$.

\section{Unperturbed orbits near the separatrix}

Consider the unperturbed Hamiltonian $H_{0}(q, p)$ near the saddle point $\left(q_{s}, p_{s}\right)$ where $\partial H_{0} / \partial q\left(q_{s}, p_{s}\right)=\partial H_{0} / \partial p\left(q_{s}, p_{s}\right)$ $=0$. Expanding $H_{0}(q, p)$ in a series of powers of $\left(q-q_{s}\right),(p$ $-p_{s}$ ) near the saddle point and by the linear coordinate transformation

$$
\begin{gathered}
\xi=\left(q-q_{s}\right) \cos \phi+\left(p-p_{s}\right) \sin \phi, \\
\eta=-\left(q-q_{s}\right) \sin \phi+\left(p-p_{s}\right) \cos \phi,
\end{gathered}
$$

the Hamiltonian $H_{0}(q, p)$ can be diagonalized:

$$
h(\xi, \eta) \equiv H_{0}(q, p)-H_{0}\left(q_{s}, p_{s}\right)=-\frac{\alpha^{2}}{2} \xi^{2}+\frac{\beta^{2}}{2} \eta^{2}+O\left(\xi^{3}, \eta^{3}\right),
$$

where $\alpha=\sqrt{\left|\lambda_{1}\right|}, \beta=\sqrt{\lambda_{2}}$, and $\lambda_{1}<0$ and $\lambda_{2}>0$,

$$
\left(\lambda_{1}, \lambda_{2}\right)=\frac{H_{q q}+H_{p p}}{2} \pm \sqrt{\frac{\left(H_{q q}-H_{p p}\right)^{2}}{4}+H_{q p}^{2}}
$$

are the eigenvalues of the matrix

$$
\left.\left(\begin{array}{ll}
H_{q q} & H_{q p} \\
H_{q p} & H_{p p}
\end{array}\right) \equiv\left(\begin{array}{cc}
\frac{\partial^{2} H_{0}}{\partial q^{2}} & \frac{\partial^{2} H_{0}}{\partial q \partial p} \\
\frac{\partial^{2} H_{0}}{\partial p \partial q} & \frac{\partial^{2} H_{0}}{\partial p^{2}}
\end{array}\right)\right|_{q=q_{s}, p=p_{s}}
$$

The angle $\phi$ is determined by $\tan \phi=2 H_{q p} /\left(H_{q q}-H_{p p}\right)$. The equations of motion in coordinates $(\xi, \eta)$ are given by

$$
\frac{d \xi}{d t}=\frac{\partial h(\xi, \eta)}{\partial \eta}=\beta^{2} \eta, \quad \frac{d \eta}{d t}=-\frac{\partial h(\xi, \eta)}{\partial \xi}=\alpha^{2} \xi
$$

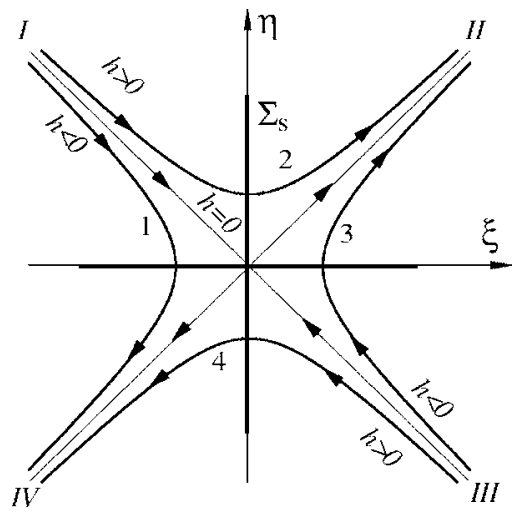

FIG. 6. Phase curves of Hamiltonian system near the saddle point.

Phase space of this system near the saddle point is shown in Fig. 6. Its solutions $[\xi(t ; h), \eta(t ; h)], 0<t<t_{s}$, which cross the section $\Sigma_{s}$ at the time moment $t \rightarrow t_{s}=\pi / \omega(h)$ along branches I and III of the separatrix are

$$
\begin{aligned}
& \xi(t ; h)=\mp \frac{\sqrt{2|h|}}{\alpha} \times \begin{cases}\cosh \left[\gamma\left(t_{s}-t\right)\right], & \text { for } h<0, \\
\sinh \left[\gamma\left(t_{s}-t\right)\right], & \text { for } h>0,\end{cases} \\
& \eta(t ; h)= \pm \frac{\sqrt{2|h|}}{\beta} \times \begin{cases}\sinh \left[\gamma\left(t_{s}-t\right)\right], & \text { for } h<0, \\
\cosh \left[\gamma\left(t_{s}-t\right)\right], & \text { for } h>0,\end{cases}
\end{aligned}
$$

where $\gamma=\alpha \beta$. The upper and lower signs in Eqs. (A9) and (A10) correspond to the solution along branches I and III, respectively. Similarly, solutions $[\xi(t ; h), \eta(t ; h)], 0>t>-t_{s}$, which cross the section $\Sigma_{s}$ at the time moment $t \rightarrow-t_{s}$ $=\pi / \omega(h)$ along branches II and IV of the separatrix are given by

$$
\xi(t ; h)= \pm \frac{\sqrt{2|h|}}{\alpha} \times \begin{cases}\cosh \left[\gamma\left(t_{s}+t\right)\right], & \text { for } h<0, \\ \sinh \left[\gamma\left(t_{s}+t\right)\right], & \text { for } h>0,\end{cases}
$$

$$
\eta(t ; h)= \pm \frac{\sqrt{2|h|}}{\beta} \times \begin{cases}\sinh \left[\gamma\left(t_{s}+t\right)\right], & \text { for } h<0, \\ \cosh \left[\gamma\left(t_{s}+t\right)\right], & \text { for } h>0 .\end{cases}
$$

\section{Perturbation Hamiltonian in normal coordinates $\xi, \eta$ near the saddle points}

We expand the perturbation Hamiltonian $V(h, \tau)$ $=H_{1}(q, p)$ near the saddle point, $\left(q_{s}, p_{s}\right)$, in series of powers of $(\xi, \eta)$ :

$$
H_{1}\left(z, p_{z}\right)=a_{\xi} \xi+a_{\eta} \eta+b_{\xi \xi} \xi^{2}+b_{\xi \eta} \xi \eta+b_{\eta \eta} \eta^{2}+O\left(\delta^{3}\right),
$$

where

$$
a_{\xi}=a_{q} \cos \alpha+a_{p} \sin \alpha
$$




$$
\begin{gathered}
a_{\eta}=-a_{q} \sin \alpha+a_{p} \cos \alpha, \\
b_{\xi \xi}=b_{q q} \cos ^{2} \alpha+\frac{1}{2} b_{z p} \sin 2 \alpha+b_{p p} \sin ^{2} \alpha, \\
b_{\xi \eta}=-b_{q q} \sin 2 \alpha+b_{q p} \cos 2 \alpha+b_{p p} \sin 2 \alpha, \\
b_{\eta \eta}=b_{q q} \sin ^{2} \alpha-\frac{1}{2} b_{q p} \sin 2 \alpha+b_{p p} \cos ^{2} \alpha .
\end{gathered}
$$

Here $\delta \sim\left|q-q_{s}\right|,\left|p-p_{s}\right|$. The coefficients $a_{q}, \ldots, b_{p p}$ are given by

$$
\begin{gathered}
a_{q}=\left.\frac{\partial H_{n}(q, p)}{\partial q}\right|_{q=q_{s}, p=p_{s}, \quad a_{p}=\left.\frac{\partial H_{n}(q, p)}{\partial p_{q}}\right|_{q=q_{s}, p=p_{s}}} \\
b_{q q}=\left.\frac{1}{2} \frac{\partial^{2} H_{n}(q, p)}{\partial q^{2}}\right|_{q=q_{s}, p=p_{s},} b_{q p}=\frac{\partial^{2} H_{n}(q, p)}{\partial q \partial p} \\
b_{p p}=\left.\frac{1}{2} \frac{\partial^{2} H_{n}(q, p)}{\partial p^{2}}\right|_{q=q_{s}, p=p_{s}}
\end{gathered}
$$

\section{Integrals over the powers of orbits $\xi(t, t), \eta(t, h)$ near the separatrix}

The oscillatory parts of $R(h)$ [Eq. (A1)] are given by the integrals $F(h, t)$ taken at the values $t= \pm t_{s}, t_{s}=\pi / \omega(h)$. According to Eq. (A13) we have expressed through the integrals over the powers of orbits $\xi(t, t), \eta(t, h)$

$$
X^{(k, j-k)}=\int^{ \pm t_{s}} \xi^{k}(t ; h) \eta^{j-k}(t ; h) \exp (i \Omega t) d t,(0 \leqslant k \leqslant j) .
$$

Below we estimate the these integrals up to the second order $(j \leqslant 2)$ : Using the solutions for $\xi(t ; h), \eta(t ; h)$ near the saddle point given by Eqs. (A9) and (A10) one obtains the following expressions for $X^{(1,0)}\left(h, t_{s}\right)$ and $X^{(0,1)}\left(h, t_{s}\right)$ along branches I and III, respectively:

$$
\begin{aligned}
& X^{(1,0)}\left(h, t_{s}\right)=\mp e^{i \Omega t_{s}} \frac{\sqrt{2|h|}}{\alpha\left(\gamma^{2}+\Omega^{2}\right)} \times \begin{cases}i \Omega, & h<0 \\
\gamma, & h>0,\end{cases} \\
& X^{(0,1)}\left(h, t_{s}\right)= \pm e^{i \Omega t_{s}} \frac{\sqrt{2|h|}}{\beta\left(\gamma^{2}+\Omega^{2}\right)} \times \begin{cases}\gamma, & h<0 \\
i \Omega, & h>0 .\end{cases}
\end{aligned}
$$

Similarly, the corresponding functions along branches II and IV are given by

$$
\begin{aligned}
& X^{(1,0)}\left(h,-t_{s}\right)= \pm e^{-i \Omega t_{s}} \frac{\sqrt{2|h|}}{\alpha\left(\gamma^{2}+\Omega^{2}\right)} \times \begin{cases}i \Omega, & h<0 \\
-\gamma, & h>0\end{cases} \\
& X^{(0,1)}\left(h,-t_{s}\right)= \pm e^{-i \Omega t_{s}} \frac{\sqrt{2|h|}}{\beta\left(\gamma^{2}+\Omega^{2}\right)} \times \begin{cases}-\gamma, & h<0 \\
i \Omega, & h>0\end{cases}
\end{aligned}
$$

respectively.

The second-order integrals $X^{(2,0)}\left(h, \pm t_{s}\right), X^{(1,1)}\left(h, \pm t_{s}\right)$, $X^{(0,2)}\left(h, \pm t_{s}\right)$ along all four branches (I-IV) are given by

$$
\begin{gathered}
X^{(2,0)}\left(h, \pm t_{s}\right)=-i e^{i \Omega t_{s} \frac{2|h|}{\alpha^{2} \Omega} C_{1}(h),} \\
X^{(1,1)}\left(h, \pm t_{s}\right)=e^{i \Omega t_{s}} \frac{2|h|}{4 \gamma^{2}+\Omega^{2}}, \\
X^{(0,2)}\left(h, \pm t_{s}\right)=i e^{i \Omega t_{s} \frac{2|h|}{\beta^{2} \Omega} C_{2}(h),}
\end{gathered}
$$

where

$$
\begin{aligned}
& C_{1}(h)= \begin{cases}\left(1+\frac{\Omega^{2}}{4 \gamma^{2}+\Omega^{2}}\right), & h<0 \\
\left(-1+\frac{\Omega^{2}}{4 \gamma^{2}+\Omega^{2}}\right), & h>0,\end{cases} \\
& C_{2}(h)= \begin{cases}\left(-1+\frac{\Omega^{2}}{4 \gamma^{2}+\Omega^{2}}\right), & h<0 \\
\left(1+\frac{\Omega^{2}}{4 \gamma^{2}+\Omega^{2}}\right), & h>0 .\end{cases}
\end{aligned}
$$

\section{Oscillatory parts of $R(h)$}

According to the relations (A3) these quantities are expressed through the functions $F\left(h, \pm t_{s}\right)$ [Eq. (A4)], which using the expansion (A13) can be reduced to

$$
\begin{aligned}
F(h, t)= & \int V(h, t) e^{i \Omega t} d t \\
= & a_{\xi} X^{(1,0)}(h, t)+a_{\eta} X^{(0,1)}(h, t)+b_{\xi \xi} X^{(2,0)}(h, t) \\
& +b_{\xi \eta} X^{(1,1)}(h, t)+b_{\eta \eta} X^{(0,2)}(h, t)+O\left(\delta^{3}\right) .
\end{aligned}
$$

First we consider separately the integrals along each branch, I-IV, of the separatrix.

\section{a. First (I) and third (III) branches}

Using the relations for the integrals $X^{(k, j-k)}$ given by Eqs. (A16), we obtain the following expressions for $R^{(o s c)-}(h)$ and its real and imaginary parts, $K^{(o s c)-}(h)$ and $L^{(o s c)-}(h)$ :

$$
R_{1}^{(o s c)+}(h)=F\left(h, t_{s}\right)=\sqrt{|h|} e^{i \pi \Omega / / \omega(h)} \times\left( \pm\left\{\begin{array}{ll}
-i \Omega A_{\xi}+\gamma A_{\eta}, & \text { for } h<0 \\
-\gamma A_{\xi}+i \Omega A_{\eta}, & \text { for } h>0
\end{array}\right\}+\sqrt{|h|}[C-i B(h)]\right)
$$




$$
\begin{aligned}
& K_{1}^{(o s c)+}(h)=\operatorname{Re} R^{(o s c)-}(h)= \pm \sqrt{|h|} \times \begin{cases}\Omega A_{\xi} \sin [\pi \Omega / \omega(h)]+\gamma A_{\eta} \cos [\pi \Omega / \omega(h)], & \text { for } h<0 \\
-\gamma A_{\xi} \cos [\pi \Omega / \omega(h)]-\Omega A_{\eta} \sin [\pi \Omega / \omega(h)], & \text { for } h>0\end{cases} \\
& +|h|\left[C \cos \left(\frac{\pi \Omega}{\omega(h)}\right)+B(h) \sin \left(\frac{\pi \Omega}{\omega(h)}\right)\right] \text {, } \\
& L_{1}^{(o s c)+}(h)=\operatorname{Im} R^{(\text {osc })-}(h)= \pm \sqrt{|h|} \times \begin{cases}-\Omega A_{\xi} \cos [\pi \Omega / \omega(h)]+\gamma A_{\eta} \sin [\pi \Omega / \omega(h)], & \text { for } h<0, \\
-\gamma A_{\xi} \sin [\pi \Omega / \omega(h)]+\Omega A_{\eta} \cos [\pi \Omega / \omega(h)], & \text { for } h>0\end{cases} \\
& +|h|\left[C \sin \left(\frac{\pi \Omega}{\omega(h)}\right)+B(h) \cos \left(\frac{\pi \Omega}{\omega(h)}\right)\right] .
\end{aligned}
$$

The coefficients $A_{\xi}, A_{\eta}, B_{1}, B_{2}$, and $C$ are defined by

$$
\begin{gathered}
A_{\xi}=\frac{\sqrt{2} a_{\xi}}{\alpha\left(\gamma^{2}+\Omega^{2}\right)}, \quad A_{\eta}=\frac{\sqrt{2} a_{\eta}}{\beta\left(\gamma^{2}+\Omega^{2}\right)}, \quad B(h)=\frac{2}{\Omega^{2}}\left(-b_{\eta \eta} \frac{C_{1}(h)}{\alpha^{2}}+b_{\xi \xi} \frac{C_{2}(h)}{\beta^{2}}\right)= \begin{cases}B_{1}, & \text { for } h<0, \\
B_{2}, & \text { for } h>0,\end{cases} \\
B_{1}=\frac{2}{\Omega^{2}}\left[\frac{b_{\xi \xi}}{\alpha^{2}}-\frac{b_{\eta \eta}}{\beta^{2}}+\frac{\Omega^{2}}{4 \gamma^{2}+\Omega^{2}}\left(\frac{b_{\xi \xi}}{\alpha^{2}}+\frac{b_{\eta \eta}}{\beta^{2}}\right)\right], \quad B_{2}=\frac{2}{\Omega^{2}}\left[-\frac{b_{\xi \xi}}{\alpha^{2}}+\frac{b_{\eta \eta}}{\beta^{2}}+\frac{\Omega^{2}}{4 \gamma^{2}+\Omega^{2}}\left(\frac{b_{\xi \xi}}{\alpha^{2}}+\frac{b_{\eta \eta}}{\beta^{2}}\right)\right], \\
C=\frac{2 b_{\xi \eta}}{4 \gamma^{2}+\Omega^{2}} .
\end{gathered}
$$

\section{b. Second (II) and fourth (IV) branches}

Using Eqs. (A17) one obtains the following expressions for $R^{(o s c)+}(h), K^{(o s c)+}(h)$, and $L^{(o s c)+}(h)$ :

$$
R_{1}^{(o s c)-}(h)=-F\left(h,-t_{s}\right)=-\sqrt{|h|} e^{-i \pi \Omega / / \omega(h)} \times\left( \pm\left\{\begin{array}{cc}
i \Omega A_{\xi}-\gamma A_{\eta}, & \text { for } h<0 \\
-\gamma A_{\xi}+i \Omega A_{\eta}, & \text { for } h>0
\end{array}\right\}+\sqrt{|h|}[C-i B(h)]\right) .
$$

The corresponding integrals are

$$
\begin{aligned}
& K_{1}^{(o s c)-}(h)=\operatorname{Re} R^{(o s c)-}(h)=\mp \sqrt{|h|} \times \begin{cases}\Omega A_{\xi} \sin [\pi \Omega / \omega(h)]-\gamma A_{\eta} \cos [\pi \Omega / \omega(h)], & \text { for } h<0 \\
-\gamma A_{\xi} \cos [\pi \Omega / \omega(h)]+\Omega A_{\eta} \sin [\pi \Omega / \omega(h)], & \text { for } h>0\end{cases} \\
& +|h|\left[-C \cos \left(\frac{\pi \Omega}{\omega(h)}\right)+B(h) \sin \left(\frac{\pi \Omega}{\omega(h)}\right)\right] \text {, } \\
& L_{1}^{(o s c)-}(h)=\operatorname{Im} R^{(o s c)+}(h)=\mp \sqrt{|h|} \times \begin{cases}\Omega A_{\xi} \cos [\pi \Omega / \omega(h)]+\gamma A_{\eta} \sin [\pi \Omega / \omega(h)], & \text { for } h<0, \\
\gamma A_{\xi} \sin [\pi \Omega / \omega(h)]+\Omega A_{\eta} \cos [\pi \Omega / \omega(h)], & \text { for } h>0,\end{cases} \\
& +|h|\left[C \sin \left(\frac{\pi \Omega}{\omega(h)}\right)-B(h) \cos \left(\frac{\pi \Omega}{\omega(h)}\right)\right] \text {. }
\end{aligned}
$$

The expressions for the integrals $R^{(o s c)}(h)$ depend on the saddle-saddle connection and can be obtained through the integrals $R^{(o s c) \pm}(h)$ given above. Below we consider the four types of homoclinic saddle-saddle connections when the system has a single hyperbolic fixed point: (i) branch II is connected with branch I of the separatrix; (ii) branch IV is connected with branch I; (iii) branch II is connected with branch III; (iv) branch IV is connected with branch III (see Fig. 6).

Case $(i)$. Adding the expressions for $R^{(o s c)+}(h)$ and $R^{(o s c)-}(h)$ taken with the upper signs we have

$$
\begin{aligned}
K^{(o s c)}(h) & =\operatorname{Re} R^{(o s c)}(h)=K^{(o s c)+}(h)+K^{(o s c)-}(h) \\
& =2 \times \begin{cases}\sqrt{|h|} A_{\eta} \gamma \sin [\pi \Omega / \omega(h)]+|h| B_{1} \sin [\pi \Omega / \omega(h)], & \text { for } h<0, \\
-\sqrt{|h|} A_{\eta} \Omega \cos [\pi \Omega \omega(h)]+|h| B_{2} \sin [\pi \Omega / \omega(h)], & \text { for } h>0,\end{cases} \\
L^{(o s c)}(h) & =\operatorname{Im} R^{(o s c)}(h)=L^{(o s c)+}(h)+L^{(o s c)-}(h) \\
& =2 \times \begin{cases}\sqrt{|h|} A_{\xi} \Omega \cos [\pi \Omega / \omega(h)]+C|h| \sin [\pi \Omega / \omega(h)], & \text { for } h<0, \\
\sqrt{|h|} A_{\xi} \gamma \sin [\pi \Omega / \omega(h)]+C|h| \sin [\pi \Omega / \omega(h)], & \text { for } h>0,\end{cases}
\end{aligned}
$$


Case (ii). Adding $R^{(o s c)+}(h)$ with the upper signs to $R^{(o s c)-}(h)$ taken with the lower signs one obtains

$$
\begin{gathered}
K^{(o s c)}(h)=2 \times \begin{cases}\sqrt{|h|} A_{\xi} \Omega \sin [\pi \Omega / \omega(h)]+|h| B_{1} \sin [\pi \Omega / \omega(h)], & \text { for } h<0, \\
-\sqrt{|h|} A_{\xi} \gamma \cos [\pi \Omega \omega(h)]+|h| B_{2} \sin [\pi \Omega / \omega(h)], & \text { for } h>0,\end{cases} \\
L^{(\text {osc })}(h)=2 \times \begin{cases}\sqrt{|h|} A_{\eta} \gamma \sin [\pi \Omega / \omega(h)]+C|h| \sin [\pi \Omega / \omega(h)], & \text { for } h<0, \\
\sqrt{|h|} A_{\eta} \Omega \cos [\pi \Omega / \omega(h)]+C|h| \sin [\pi \Omega / \omega(h)], & \text { for } h>0 .\end{cases}
\end{gathered}
$$

In case (iii) the formulas for $K^{(o s c)}(h)$ and $L^{(o s c)}(h)$ are given by Eqs. (A31) and (A32), respectively, taken with the opposite signs, and in case (iv) $K^{(o s c)}(h)$ and $L^{(o s c)}(h)$ are obtained from Eqs. (A29) and (A30) by changing the sign.

[1] N. N. Filonenko and G. M. Zaslavsky, Zh. Eksp. Teor. Fiz. 54, 1590 (1968) [Sov. Phys. JETP 27, 851 (1968)].

[2] B. V. Chirikov, Phys. Rep. 52, 265 (1979).

[3] A. J. Lichtenberg and M. A. Lieberman, Regular and Stochastic Motion, 2nd Ed. (Springer-Verlag, New York, 1992).

[4] G. M. Zaslavsky, R. Z. Sagdeev, D. A. Usikov, and A. A. Chernikov, Weak Chaos and Quasi-regular Patterns (Cambridge University Press, Cambridge, England, 1991).

[5] V. K. Melnikov Trans. Mosc. Math. Soc. 12, 1 (1963).
[6] S. S. Abdullaev, Phys. Rev. E 70, 046202 (2004).

[7] S. S. Abdullaev, J. Phys. A 35, 2811 (2002).

[8] S. S. Abdullaev, Phys. Rev. E 62, 3508 (2000).

[9] S. S. Abdullaev, and G. M. Zaslavsky, Phys. Plasmas 2, 4533 (1995).

[10] S. S. Abdullaev, and G. M. Zaslavsky, Phys. Plasmas 3, 516 (1996).

[11] R. McLachlan and P. Atela, Nonlinearity 5, 541 (1992). 